\title{
Emerging and expanding streamer head in low-pressure air
}

\author{
T. Hoder ${ }^{1,2}$, Z. Bonaventura ${ }^{2}$, V. Prukner ${ }^{1}$, \\ F.J. Gordillo-Vázquez ${ }^{3}$, M. Šimek ${ }^{1}$ \\ ${ }^{1}$ Institute of Plasma Physics v.v.i., Department of Pulse Plasma Systems, Academy \\ of Sciences of the Czech Republic, Prague, Czech Republic \\ 2 Department of Physical Electronics, Faculty of Science, Masaryk University, Brno, \\ Czech Republic \\ ${ }^{3}$ Instituto de Astrofísica de Andalucía (IAA-CSIC), Glorieta de la Astronomía s/n, \\ Granada, Spain \\ E-mail: hoder@physics.muni.cz
}

\begin{abstract}
The emergence of a streamer from an ionisation wave and its expansion are ultra-fast processes shaping the very first moments of the streamer development, and are usually accessible only by complex numerical models. In this Letter, we report experimental evidence of the emergence of a streamer from an ionisation wave in $1.3 \mathrm{kPa}$ air, a laboratory analogue of early-stage streamers emerging in geophysical Blue Starters and Jets. The radially and temporally resolved electric field patterns of an expanding streamer are determined by sub-nanosecond optical emission spectroscopy. As the emerged streamer expands, the electric field decreases by a factor of $1.4 \mathrm{in}$ $1 \mathrm{~ns}$. We quantify the radial expansion of the streamer head and its axial acceleration, reaching the velocity of $10^{7} \mathrm{~m} / \mathrm{s}$. In combination with electrical measurements, the transferred charge, electron density, and mean electron energy are quantified, enabling detailed insight into this ultra-fast phenomenon at its characteristic time-scale.
\end{abstract}

PACS numbers: 52.70.-m, 52.40.Hf, 52.80.-s

Keywords: streamer, electric field, air, electron density, low pressure, ionisation wave, Blue Jet, Blue Starter, transient luminous events

Submitted to: Plasma Sources Sci. Technol. 
Ionisation in atmospheric air frequently takes the form of contracted discharge filaments ruled by the streamer mechanism $[1,2,3,4,5,6]$. Streamers are ultra-fast contracted ionisation waves of enhanced electric field producing high-energy electrons and excited and ionised species [7]. Electron driven processes then change the chemical composition of the gas around the running streamer head. This fact is of crucial importance anywhere the streamer mechanism occurs: ozone generation and surface treatment using barrier and corona discharges [8, 9], as well as the plasma chemistry generated by streamers in lightning or in transient luminous events (TLEs, i.e. Sprites, Blue Starters and Jets etc.) [10, 11, 12]. A profound understanding of the effects of atmospheric electricity $[10,13,12,14]$ or of the streamer-induced air chemistry in various applications $[8,15]$ relies on a detailed knowledge of the magnitude of the electric field in the streamer head.

While the quasi-stationary phase of a development of a non-branching streamer in free space is a well understood phenomenon (i.e. $[16,17,18,19]$ ), the very first stages of the initiation of the streamer in pulse driven/triggered discharges is still an unresolved issue. The general mechanism of the emergence of a streamer from a local instability or irregularity in the effective ionisation rate (due to perturbations in the background electron density or electric field redistribution) is an important issue for the physics of transient laboratory discharges [8, 20, 21, 22, 23] as well as for electrical phenomena in the upper atmosphere $[24,25,26,27,28]$. Different models describe various effects and propose solutions which have not been validated experimentally. This is due to the typically high velocity of the streamer and to its erratic appearance, both in upper atmosphere luminous events as well as in large scale ozone generators or plasma treatment devices in the laboratory. Both theoretical $[20,18,17,29,30,7]$ and experimental [31, 32, 21, 19, 33] efforts have been made to understand this extremely fast ionisation process and to describe its development by the quantification of the electric field under different conditions. However, its detailed analysis from the initial conditions to the self-generated electric field enhancement in the contracted streamer head and its expansion remains mainly theoretical [20, 34, 26, 35, 36]. For example, Luque et al. [26], Liu et al. [37, 38] and others have theoretically shown that a downward-propagating ionisation wave collapses into a Sprite streamer as it propagates farther down under conditions in the lower ionosphere. We believe that the laboratory analogues of streamers in such discharges, as presented here, are of great interest for a deeper understanding of the studied phenomena.

In lower pressure pulse-initiated discharges, the ionisation starts with an initial diffuse ionisation cloud rapidly being transformed into an ionisation wave propagating with velocities lower than a typical streamer velocity [22, 26, 39]. Under certain conditions (see $[22,40,41]$ ) this wave collapses and transforms into a contracted ionisation wave, called a streamer, which has the form of a thin discharge filament. An analogous situation was recently simulated based on different sources of ionisation enhancement $[20,26,28,37,38]$. These results show how the very thin streamer emerges at first and subsequently expands until it reaches the quasi-stationary phase under 
given conditions. This very first phase of the early-stage streamer development remains experimentally unresolved. It is usually believed that pulsed corona discharges are the closest laboratory analogue for TLE streamers [11, 42] (although other approaches have been investigated as well, e.g. [43]). Such investigations have resulted in the quantification of the average magnitudes of crucial streamer parameters (velocity, diameter etc.). However, the irreproducibility of such pulsed discharges and of the real TLE events hinders spectroscopic studies with high spatiotemporal resolution and makes any deeper analysis impossible. In particular, an understanding of the emergence of the streamer from the ionisation wave is experimentally based only on averaged imaging, see, e.g. [22]. As a result, the only information we have is the fact that at the moment of the emergence of the streamer its diameter is much lower than typically expected for the given conditions, compare the figures presented in [22, 40, 41] and recordings of real geophysical events [28, 37]. Early-stage streamers generated in dielectric barrier discharges are reproducible as well as stable [33, 44] even though the full development of the streamer is partially limited by Townsend scaling and the confinement of the discharge chamber.

The determination of the electric field in small-scale highly-transient plasmas is a challenging task, as direct measurements using laser spectroscopy still lack the necessary high-resolution and sensitivity [45]. Even though new methods have emerged [46, 47], the optical emission spectroscopy based method, as recently further developed by coauthors of this Letter $[19,33,48,49,50,51]$, remains usually the only option. In this Letter, we present unique experimental results on expanding non-branching positive streamers in low pressure air. We present a detailed investigation of the very first moments of the initiation of the streamer, i.e. before the streamer achieves its quasistationary state. The investigated early-stage streamers are laboratory analogues to those present in the Blue Starters and Blue Jets observed in the lower stratosphere $[52,53]$. We choose the volume barrier discharge setup in which reproducible and stable streamer events are generated periodically. This allows tracking the streamer initiation process from its very origin. We reveal an emerging streamer and describe its expansion by the experimentally determined electric field. This is the first experimental evidence of elementary streamer stages expressed quantitatively via the electric field with a resolution enabling a complete insight.
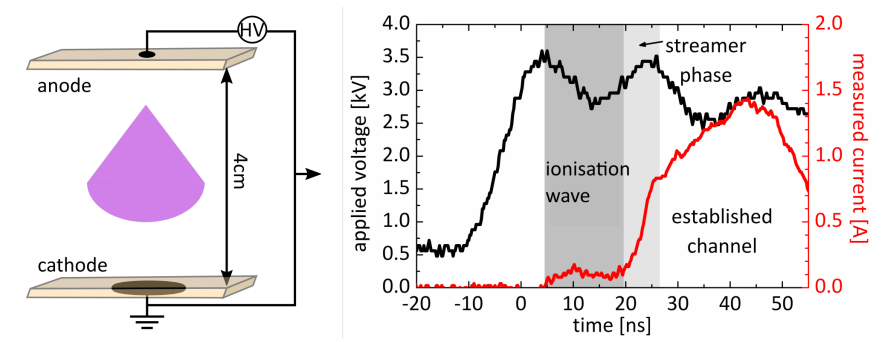

Figure 1. Simplified scheme of the experimental setup (left) and the measured electrical parameters in the external circuit. 
For the experiment, we selected the pressure of $1.3 \mathrm{kPa}$ to imitate the midstratospheric conditions at an altitude of approximately $25 \mathrm{~km}$. The discharge was generated in synthetic air between two electrodes (point anode and circle-shaped cathode, both covered by alumina dielectrics) with an inter-electrode gap of $d=$ $4 \mathrm{~cm}$, see Fig. 1 and in Ref. [33]. The barrier discharge electrode system was placed in vacuum inside a stainless steel chamber. The barrier discharge arrangement allowed the stabilisation of the occurrence of the streamer both temporally and spatially, which enabled a long-lasting accumulation of weak light emission of the streamer. A schematic drawing of the investigated discharge structure and the way it was radially scanned by a photomultiplier (PMT) is shown in Fig. 2. The optical emission was recorded by PMT (Hamamatsu H10721, rise-time of $570 \mathrm{ps}$ ) via $25 \mathrm{~cm}$ long capillary with an inner diameter of $1 \mathrm{~mm}$. In order to record only spectrally selected emissions of the first negative and second positive system band, a set of suitable bandpass filters was applied. For the signal of the second positive system (SPS) with 0-0 vibrational transition, band head at $337.1 \mathrm{~nm}$, a bandpass filter with FWHM of $9.7 \mathrm{~nm}$ was used (LOT Oriel 337FS10). For the $391.5 \mathrm{~nm}$ wavelength, a Semrock FF01-395/11 filter (central wavelength of $395 \mathrm{~nm}$, FWHM $16.1 \mathrm{~nm}$ ) was used, and for $370 \mathrm{~nm}$, a Semrock FF01-370/10 filter (central wavelength $370 \mathrm{~nm}$, FWHM $11.5 \mathrm{~nm}$ ). The emission FNS waveform was corrected due to the overlap with the tail of the SPS $(2,5)$ band, which was done using spectra simulation as described in [33]. The recorded spectrally resolved waveforms were fitted and an Abel inversion procedure was applied to obtain the local intensity of the cylindrically symmetric discharge event.

In order to be able to trigger the streamer discharge with sufficient precision for an accurate PMT-scanning, a complex applied high-voltage waveform was used. Namely, an AC burst mode was employed with repetition frequency of $30 \mathrm{~Hz}$. In the burst, two subsequent sine waveforms with a frequency of $1 \mathrm{kHz}$ and amplitude of $3 \mathrm{kV}$ were superimposed with a 500-ns long high-voltage pulse with an amplitude of $2.5 \mathrm{kV}$ and rise time of $10 \mathrm{~ns}$. The stability of the current and voltage waveforms was monitored using high-bandwidth current (Pearson 2877) and high-voltage (Tektronix P6015A) probes respectively.

The measured applied voltage and the current in the external circuit are shown in Fig. 1. Three basic phases characteristic of the streamer discharge [20, 33] under given conditions can be identified: the primary ionisation and the formation of the diffuse (not contracted) ionisation wave is responsible for the first increase of the current. The subsequent rapid current rise tracks the positive streamer emergence and propagation. Lastly, as the streamer impacts onto the cathode dielectrics at approximately $\mathrm{t}=27 \mathrm{~ns}$, the transient conductive plasma channel is created. The fast deposition of the generated electric charge onto the dielectrics then quenches the discharge in the next $100 \mathrm{~ns}$. In this Letter, the dynamics of the ionisation wave and mainly the early-stage streamer emergence and propagation (see also in Fig. 3) are studied in detail.

In Fig. 3a), the vertical scan of the total discharge luminosity development is shown. The inter-electrode gap was scanned with steps of $1 \mathrm{~mm}$ by the high- 


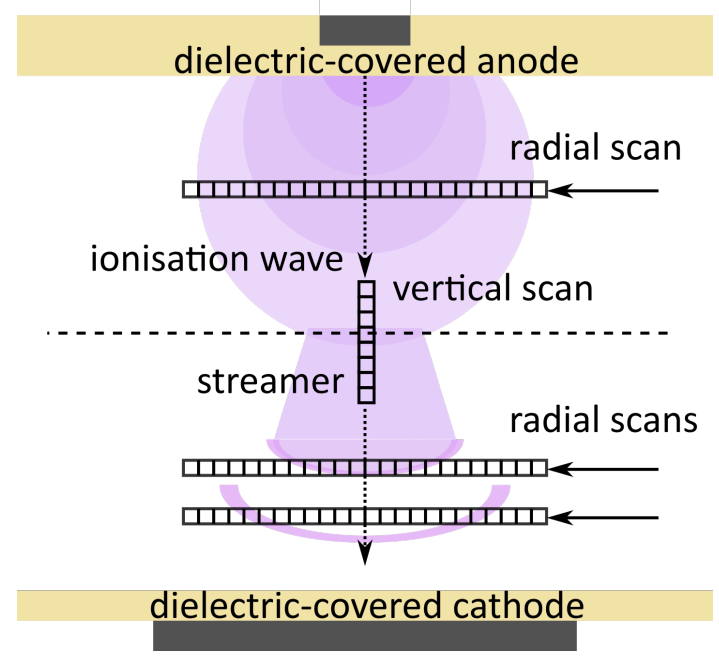

Figure 2. Schematic drawing of the DBD-electrode geometry, discharge pattern and the scanning procedures.

speed photomultiplier, which was correlated with the applied voltage waveform. The above mentioned two important phases are denoted with coloured arrows. From the spatiotemporal development, the velocity of the diffuse ionisation wave, $1.5 \times 10^{6} \mathrm{~m} / \mathrm{s}$, and the velocity of the streamer, $1.5 \times 10^{7} \mathrm{~m} / \mathrm{s}$, were determined and are coherent with theoretically obtained values of the ionisation wave and early-stage streamer phenomena, see Ref. [26, 54].

Panel b) of Fig. 3 shows the results of an electrical analysis of the current and voltage signals measured in an external circuit. The intrinsic electrical characteristics of the discharge plasma were inferred using methods presented in Ref. [55, 33]. The resulting development of the resistance of the plasma, of the effective electric field in the gap, and of the net transferred charge, are shown in Fig. 3b). The resistance was computed from the following equation (see the analysis in Ref. [55]):

$$
\varrho(t)=\frac{U_{g}(t)}{j_{R}(t)}=\frac{V(t)-Q(t) / C_{d}}{\left(1-\frac{C_{c e l l}}{C_{d}}\right)^{-1}\left(i(t)-C_{c e l l} \frac{\mathrm{d} V(t)}{\mathrm{d} t}\right)}
$$

where $j_{R}(t)$ is the net discharge current, $U_{g}(t)$ is the gap voltage, $i(t)$ is the measured current, $V(t)$ is the measured applied voltage in the external circuit, $Q(t)$ is the charge transferred in the external circuit, i.e. the integral of $i(t)$, while $C_{c e l l}=1.6 \mathrm{pF}$ and $C_{d}$ $=18 \mathrm{pF}$ are the effective capacitances of the discharge system determined by adopting the approach of Pipa et al. [55].

Apparently, the resistance drops rapidly during the development of the first ionisation in the gap and subsequently also during the propagation of the streamer and its impact on the dielectric surface. In total, it drops by over three orders of magnitude. Within the first $40 \mathrm{~ns}$, a charge of $20 \mathrm{nC}$ is transferred through the gap (as shown in Fig. 3), finally it is $70 \mathrm{nC}$ by the discharge in total. The ionisation wave transfers approximately $1.4 \mathrm{nC}$ during the first $20 \mathrm{~ns}$ until its transformation into the 
a)

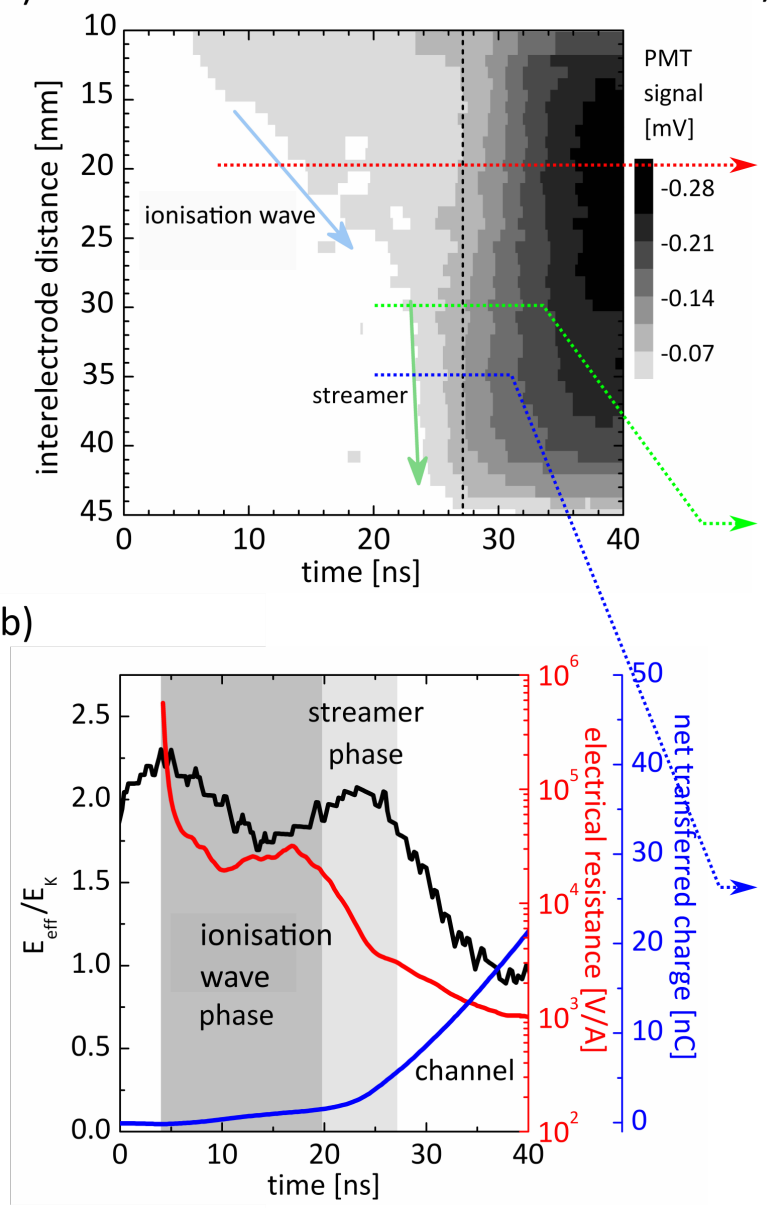

c)
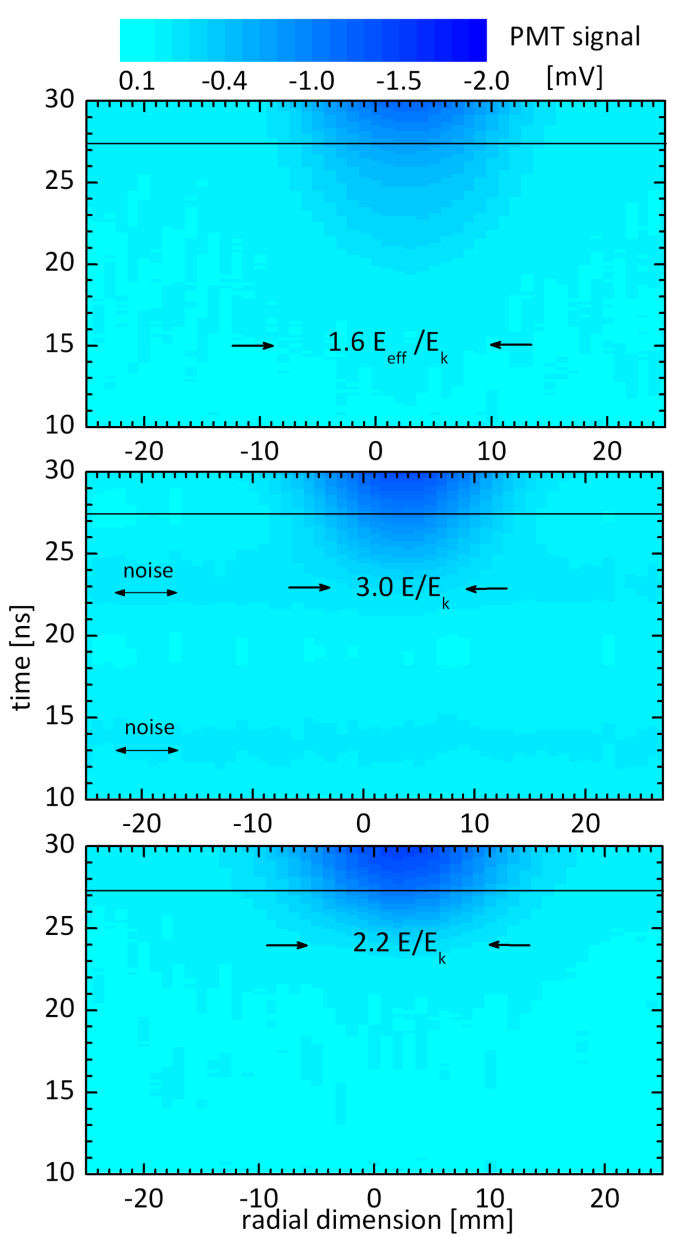

Figure 3. The axial (a) and radial (c) scans of the line-of-sight integrated discharge light emission as recorded with 570 ps resolution photomultiplier and sampled each $200 \mathrm{ps}$. In panel (b), the instantaneous development of the gas resistance (red), the effective electric field (black) and net transferred charge (blue) in the discharge are shown. The value of $E_{\mathrm{k}}$ is taken as $120 \mathrm{Td}$ for given gas density at pressure $1.3 \mathrm{kPa}$ (10 torr) and temperature of $300 \mathrm{~K}$. The arrows in panel c) mark the front of the given ionisation event, wave (up) or streamer (middle, bottom). The repetition frequency of the high-voltage pulse, together with the sinusoidal waveform burst, was $30 \mathrm{~Hz}$.

early-stage streamer. This value corresponds to a number of electrons on the order of $10^{9}$, which corresponds to a density of $2 \cdot 10^{15} \mathrm{~m}^{-3}$ for the observed centimetre dimensions. Clearly, the criterion for streamer initiation is locally achieved under given conditions and the electron multiplication grows rapidly [20, 56]. At that moment, the diffuse ionisation wave becomes unstable and the early-stage streamer emerges. Between the 20 th and 27th nanoseconds, the early-stage streamer generates a charge of $3.6 \mathrm{nC}$. The effective electric field in the gap $E_{\text {eff }}=U_{g}(t) / d$ is expressed relatively to the threshold value $E_{k}$ for which the air ionisation equals the electron attachment $\alpha=\eta$, i.e. $E_{k}=$ $120 \mathrm{Td}$ (i.e. $32 \mathrm{kV} / \mathrm{cm}$ at atmospheric pressure). During the ionisation wave, the mean electric field in the gap reaches $E_{e f f}=1.6 E_{k}$. Note that this is a value determined 
from the electric measurement and therefore is an averaged value over the whole gap. The FNS emission at this coordinate was too weak compared with the SPS emission for a proper spectroscopic determination of the local electric field. Nevertheless, such a weak FNS intensity clearly provides complementary spectroscopic evidence of a very weak local E/N [48, 49].

In panel c) of Fig. 3, the radial scans of the total discharge luminosity at three vertical coordinates $y$ are shown. The arrow with the peak electric field value denotes the position of the ionisation wave front ( $15 \mathrm{~ns}$, first radial scan) and the front of the streamer head (prior to and after the 23th nanoseconds at second and third scan, respectively) inferred from sub-nanosecond spectroscopy, see Ref.[19] and further in the text. The horizontal black line at approximately $27 \mathrm{~ns}$ denotes the moment of the impact of the streamer onto the cathode. It is apparent from the luminosity profiles at the denoted times that the ionisation wave, and later the streamer head, expands. The velocity of its radial expansion, in the range of $10^{6} \mathrm{~m} / \mathrm{s}$, is approximately one order of magnitude lower than the propagation velocity of the streamer head in the axial direction, as has been recently shown in Ref. [44]. Here both these velocities are also faster than in Ref. [44], which is caused by the more efficient photoionisation in the mixture with higher oxygen content [57] and lower pressure [58] in present case.

Using spectral band-pass filters, the spatially resolved emission waveforms of the emerging streamer event were recorded for selected nitrogen spectral bands. The line-ofsight radially resolved emission distribution was found to be Gaussian, and the process of Abel deconvolution described in Refs. [59, 33] was used to obtain the local emission of the streamer front. The radially resolved local emission intensities of the radiative states $\mathrm{N}_{2}\left(\mathrm{C}^{3} \Pi_{u}\right)$ (second positive system SPS with $(0,0)$ vibronic transition at $337.1 \mathrm{~nm}$ ) and $\mathrm{N}_{2}^{+}\left(\mathrm{B}^{2} \Sigma_{u}^{+}\right)$(first negative system FNS with $(0,0)$ vibronic transition at $391.5 \mathrm{~nm}$ ) were obtained from the corresponding line-of-sight waveforms. The differential form of the ratio of these intensities is strongly dependent on the local electric field $[9,33,48]$ :

$$
\frac{\tau_{\text {eff }}^{\mathrm{FNS}} \cdot \mathrm{d} I_{\mathrm{FNS}} / \mathrm{d} t+I_{\mathrm{FNS}}}{\tau_{\text {eff }}^{\mathrm{SPS}} \cdot \mathrm{d} I_{\mathrm{SPS}} / \mathrm{d} t+I_{\mathrm{SPS}}}=R_{\mathrm{FNS} / \mathrm{SPS}}(E / N)
$$

where $I_{\mathrm{FNS}}$ and $I_{\mathrm{SPS}}$ denote the measured intensities of FNS and SPS, respectively, and $\tau_{\text {eff }}^{F N S}=3.05 \mathrm{~ns}$ and $\tau_{\text {eff }}^{S P S}=20.54 \mathrm{~ns}$ are the effective lifetimes for the pressure being considered which were obtained from the same constants as in [33]. The dependence $R_{\mathrm{FNS} / \mathrm{SPS}}(E / N)$ was given by the following equation:

$$
R_{F N S / S P S}(E / N)=0.5 \cdot 46 \cdot 0.065 \cdot \exp \left[-89(E / N)^{-0.5}-402(E / N)^{-1.5}\right],
$$

see $[48,49,33]$. The obtained spatiotemporal distributions of the reduced electric field strength for the emerging and for the expanding streamer are shown in Fig. 4. The determined electric field data were extrapolated to the lower field values (under the value of $15 \%$ of waveform maximum) by fitting the electric field waveform using the analytic expression presented by Kulikovsky in Refs. [60].

It is apparent from Fig. 4 that the curvature of the just emerged streamer head, 


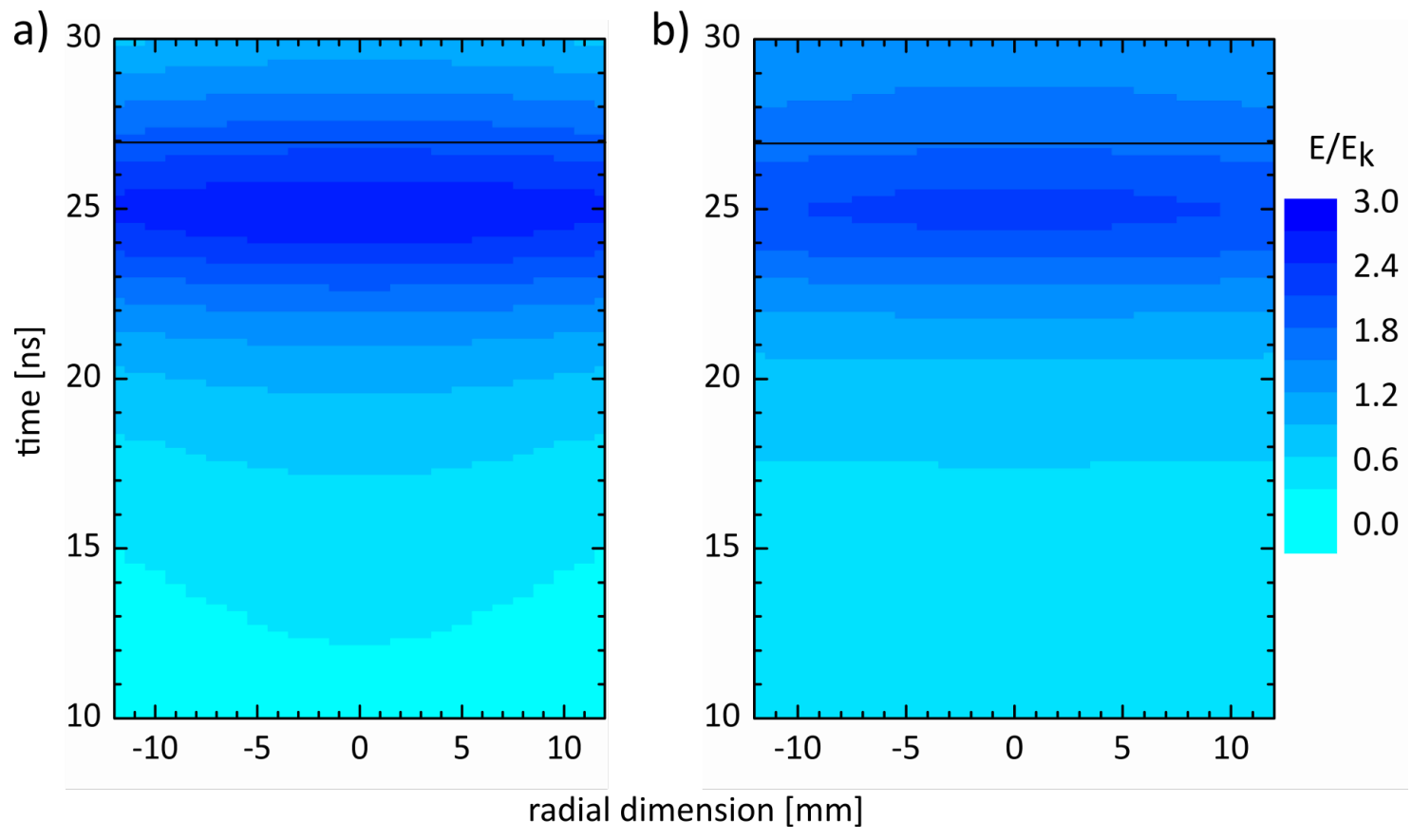

Figure 4. Determined electric field in the emerging a) and subsequently expanding b) streamer head. The enhanced local electric field is given with respect to $E_{\mathrm{k}}=$ $120 \mathrm{Td}$. The repetition frequency of the high-voltage pulse, together with the sinusoidal waveform burst, was $30 \mathrm{~Hz}$ in all presented experiments.

panel a), is smaller than in the case of the already expanded streamer, panel b). Almost in $1 \mathrm{~ns}$, the streamer becomes broader and the peak value of the electric field decreases by a factor of $\sim 1.4$. This is in agreement with the theoretical results of Luque et al. [16] as well as with recent results of geophysical streamer simulations [37, 38]. Here, the peak value of the electric field decreases from almost $3 E_{k}$ for the emerging streamer stage down to $2.2 E_{k}$ for the expanding stage. We assume that the influence of the cathode charging by the approaching streamer head is negligible and so the streamer can be described as propagating in free space at the given coordinate.

Furthermore, to quantify the electron density, we have adopted the approach using the determined discharge current and local electric field (similarly to Ref. [61]). The fundamental parameter $n_{e}(t)$ was computed for the emerging streamer using the following equation:

$$
n_{e}(t)=\frac{1}{e \mu_{e}(E(t)) E(t)}\left(\frac{j_{R}(t)}{A}-\varepsilon_{0} \frac{\partial E(t)}{\partial t}\right)
$$

where $\mu_{e}(E(t))$ is the local electron mobility dependent on the temporally changing electric field, $j_{R}(t)$ is the net discharge current, and $A$ is the local cross-sectional area of the filament given by its diameter, which was obtained from measurements similar to those presented in Fig. 3c). This one-dimensional approach may overestimate $n_{e}(t)$ under the given conditions. The dependence of the electron mobility on the determined electric fields was computed using the two-term approximation to the solution of the 
Boltzmann equation and the Biagi cross-sections [62, 63]. Furthermore, the development of the mean electron energy was determined from the same computations.

The results for the streamer-initiated electron ensemble parameters are shown in Fig. 5. After the 20th nanosecond, the current is rising rapidly. Due to the positive value of $\partial E / \partial t$, the resulting increase in the electron density is a bit decelerated (see Eq.4), but finally reaches the value of $1 \cdot 10^{16} \mathrm{~m}^{-3}$ at the moment of the maximum value of the electric field, i.e. in the streamer head. Subsequently, as the current rise decelerates, the further growth of the electron density is almost stopped at $26 \mathrm{~ns}$. Later on, the term $\partial E / \partial t$ gets smaller and even negative and so the rise of the electron density starts again, increasing almost one order of magnitude in the next several nanoseconds. The values found are in good agreement with the Townsend scaling (for electron density with $\sim N^{2}$, where $N$ is the air particle density) and with the theoretically obtained values in Ref. [54]. The maximum mean electron energy reaches $7.5 \mathrm{eV}$.
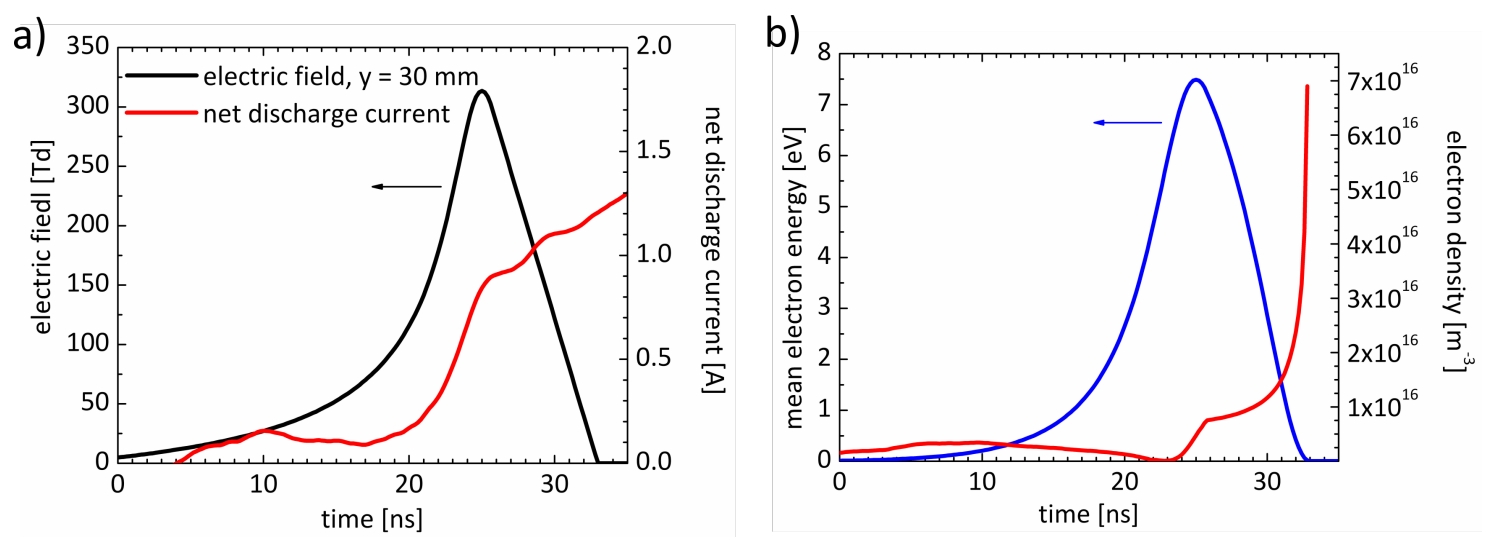

Figure 5. Instantaneous development of the electric field, net discharge current a), mean electron energy and electron density according to Eq. (4), see panel b).

In conclusion, we have reported unique results of an experimental determination of the basic plasma parameters of a streamer head in the early stages of its development when it emerges from the ionisation wave and further expands. We have quantified the radial development of the electric field, electron density, and the streamer acceleration in the axial as well as the radial direction with sub-nanosecond resolution. Our results confirm the outcomes of numerical predictions [16]. It is worth noting that these results bear a striking similarity to the numerically obtained results of streamer emergence from ionisation waves reported in $[26,28,37,38]$, yet under different pressures.

In addition, the results in this Letter have an important methodological consequence. The spectrally resolved imaging of accidentally appearing lightning events is practically the only method for obtaining spatially and temporally resolved information about lightning or TLEs streamers [64, 65, 66, 67]. As a consequence, a detailed experimental spectroscopic understanding of the TLE streamer analogues has to be obtained primarily under laboratory conditions [33, 64, 7] as has been proposed here for the streamer analogue of a Blue Starter and Jet. In this Letter we have 
taken an essential step towards revealing the microphysics of the emerging streamers on their ultra-short characteristic time-scales under conditions of pressure relevant for the TLEs. The approach presented for the determination of the electric field from sub-nanosecond recording is important not only for the investigation of atmospheric electricity phenomena at the ground level, but also for investigations carried out with balloons or satellites. The appropriately equipped ASIM [68] and TARANIS [69] spacemissions of the European Space Agency and the French Space Agency, respectively, will provide new insights into the fundamental physics of lightning and TLEs. The spacemission data [70] might be analysed in the light of laboratory benchmark experiments trying to mimic specific aspects of those phenomena, as has been presented in this Letter.

\section{Acknowledgements}

This research was funded by the Academy of Sciences of the Czech Republic under project M100431201. TH was supported by the European Science Foundation Research Networking Programme TEA-IS (ex-change grant No. 4219). ZB was supported by the Czech Science Foundation, project No. 15-04023S. FJGV was supported by the Spanish Ministry of Science and Innovation (MINECO) under project ESP2017-86263C4-4-R. TH is grateful for the support of project LM2018097 funded by the Ministry of Education, Youth and Sports of the Czech Republic.

\section{References}

[1] Raether H 1939 Zeitschrift für Physik 112 464-489 ISSN 0044-3328 URL https://doi.org/10. 1007/BF01340229

[2] Loeb L B 1948 Phys. Rev. 74(2) 210-212 URL https://link.aps.org/doi/10.1103/PhysRev. 74.210

[3] Meek J M 1940 Phys. Rev. 57(8) 722-728 URL https://link.aps.org/doi/10.1103/PhysRev. 57.722

[4] Marode E, Djermoune D, Dessante P, Deniset C, Ségur P, Bastien F, Bourdon A and Laux C 2009 Plasma Physics and Controlled Fusion 51124002 URL http://stacks.iop.org/0741-3335/ $51 / i=12 / a=124002$

[5] Naidis G V, Tarasenko V F, Babaeva N Y and Lomaev M I 2018 Plasma Sources Science and Technology 27013001 URL https://doi.org/10.1088\%2F1361-6595\%2Faaa072

[6] Černák M, Hoder T and Bonaventura Z 2019 Plasma Sources Science and Technology URL https://doi.org/10.1088/1361-6595/ab5051

[7] Šimek M and Bonaventura Z 2018 Journal of Physics D: Applied Physics 51504004 URL https://doi.org/10.1088\%2F1361-6463\%2Faadcd1

[8] Kogelschatz U 2004 Plasma Physics and Controlled Fusion 46 B63 URL http://stacks.iop. org/0741-3335/46/i=12B/a=006

[9] Hoder T, Černák M, Paillol J, Loffhagen D and Brandenburg R 2012 Physical Review E 86055401

[10] Pasko V P 2010 Journal of Geophysical Research: Space Physics 115 (Preprint https://agupubs.onlinelibrary.wiley.com/doi/pdf/10.1029/2009JA014860) URL https://agupubs.onlinelibrary.wiley.com/doi/abs/10.1029/2009JA014860

[11] Pasko V P 2007 Plasma Sources Science and Technology 16 S13-S29 URL https://doi.org/10. 
$1088 \% 2 \mathrm{~F} 0963-0252 \% 2 \mathrm{~F} 16 \% 2 \mathrm{~F} 1 \% 2 \mathrm{Fs} 02$

[12] Gordillo-Vázquez F J 2008 Journal of Physics D: Applied Physics 41234016 URL https: //doi.org/10.1088\%2F0022-3727\%2F41\%2F23\%2F 234016

[13] Pasko V P 2008 Plasma Physics and Controlled Fusion 50124050 URL https://doi.org/10. $1088 \% 2 \mathrm{~F} 0741-3335 \% 2 \mathrm{~F} 50 \% 2 \mathrm{~F} 12 \% 2 \mathrm{~F} 124050$

[14] Winkler $\mathrm{H}$ and Notholt J 2015 Journal of Atmospheric and Solar-Terrestrial Physics 122 75 - 85 ISSN 1364-6826 URL http://www.sciencedirect.com/science/article/pii/ S1364682614002430

[15] Černák M, Kováčik D, Ráhel J, Stahel P, Zahoranová A, Kubincová J, Tóth A and Černáková L 2011 Plasma Physics and Controlled Fusion 53124031 URL http://stacks.iop.org/ $0741-3335 / 53 / i=12 / a=124031$

[16] Luque A, Ratushnaya V and Ebert U 2008 Journal of Physics D: Applied Physics 41234005 URL http://stacks.iop.org/0022-3727/41/i=23/a=234005

[17] Naidis G V 2009 Phys. Rev. E 79(5) 057401 URL https://link.aps.org/doi/10.1103/ PhysRevE.79.057401

[18] Bonaventura Z, Bourdon A, Celestin S and Pasko V P 2011 Plasma Sources Science and Technology 20035012 URL http://stacks.iop.org/0963-0252/20/i=3/a=035012

[19] Hoder T, Bonaventura Z, Bourdon A and Šimek M 2015 Journal of Applied Physics 117 073302 (Preprint https://doi.org/10.1063/1.4913215) URL https://doi .org/10.1063/1. 4913215

[20] Yurgelenas Y V and Wagner H E 2006 Journal of Physics D: Applied Physics 39 4031-4043 URL https://doi.org/10.1088\%2F0022-3727\%2F39\%2F $18 \% 2 \mathrm{~F} 015$

[21] Kozlov K V and Wagner H E 2007 Contributions to Plasma Physics $47 \quad 26-33$ (Preprint https://onlinelibrary.wiley.com/doi/pdf/10.1002/ctpp.200710005) URL https://onlinelibrary.wiley.com/doi/abs/10.1002/ctpp. 200710005

[22] Clevis T T J, Nijdam S and Ebert U 2012 Journal of Physics D: Applied Physics 46045202 URL https://doi.org/10.1088\%2F0022-3727\%2F46\%2F4\%2F045202

[23] Inada Y, Aono K, Ono R, Kumada A, Hidaka K and Maeyama M 2017 Journal of Physics D: Applied Physics 50174005 URL https://doi.org/10.1088\%2F1361-6463\%2Faa65ee

[24] Wilson C T R 1924 Proceedings of the Physical Society of London 37 32D-37D URL https: //doi.org/10.1088\%2F $1478-7814 \% 2$ F37\%2F $1 \% 2 F 314$

[25] Franz R C, Nemzek R J and Winckler J R 1990 Science 249 48-51 ISSN 0036-8075 (Preprint https://science.sciencemag.org/content/249/4964/48.full.pdf) URL https: //science.sciencemag.org/content/249/4964/48

[26] Luque A and Ebert U 2009 Nature Geoscience 2757 EP - URL https://doi.org/10.1038/ ngeo662

[27] Qin J, Celestin S and Pasko V P 2011 Journal of Geophysical Research: Space Physics 116 (Preprint https://agupubs.onlinelibrary.wiley.com/doi/pdf/10.1029/2010JA016366) URL https://agupubs.onlinelibrary.wiley.com/doi/abs/10.1029/2010JA016366

[28] Qin J, Pasko V P, McHarg M G and Stenbaek-Nielsen H C 2014 Nature Communications 53740 EP - URL https://doi.org/10.1038/ncomms4740

[29] Celestin S and Pasko V P 2010 Geophysical Research Letters 37 (Preprint https: //agupubs.onlinelibrary.wiley.com/doi/pdf/10.1029/2010GL042675) URL https: //agupubs.onlinelibrary.wiley.com/doi/abs/10.1029/2010GL042675

[30] Luque A, Ebert U and Hundsdorfer W 2008 Phys. Rev. Lett. 101(7) 075005 URL https: //link.aps.org/doi/10.1103/PhysRevLett.101.075005

[31] Kuo C L, Chou J K, Tsai L Y, Chen A B, Su H T, Hsu R R, Cummer S A, Frey H U, Mende S B, Takahashi Y and Lee L C 2009 Journal of Geophysical Research: Space Physics 114 (Preprint https://agupubs.onlinelibrary.wiley.com/doi/pdf/10. 1029/2008JA013791) URL https://agupubs.onlinelibrary.wiley.com/doi/abs/10.1029/ 2008JA013791 
[32] Wagenaars E, Bowden M D and Kroesen G M W 2007 Phys. Rev. Lett. 98(7) 075002 URL https://link.aps.org/doi/10.1103/PhysRevLett.98.075002

[33] Hoder T, Šimek M, Bonaventura Z, Prukner V and Gordillo-Vázquez F J 2016 Plasma Sources Science and Technology 25045021 URL http://stacks.iop.org/0963-0252/25/i= $4 / a=045021$

[34] Liu N, Kosar B, Sadighi S, Dwyer J R and Rassoul H K 2012 Phys. Rev. Lett. 109(2) 025002 URL https://link.aps.org/doi/10.1103/PhysRevLett.109.025002

[35] Pechereau F, Bonaventura Z and Bourdon A 2016 Plasma Sources Science and Technology 25 044004 URL https://doi.org/10.1088\%2F0963-0252\%2F25\%2F4\%2F044004

[36] Dubinova A, Rutjes C, Ebert U, Buitink S, Scholten O and Trinh G T N 2015 Phys. Rev. Lett. 115(1) 015002 URL https://link.aps.org/doi/10.1103/PhysRevLett.115.015002

[37] Liu N, Dwyer J R, Stenbaek-Nielsen H C and McHarg M G 2015 Nature Communications 67540 EP - URL https://doi.org/10.1038/ncomms8540

[38] Liu N, Boggs L D and Cummer S A 2016 Geophysical Research Letters 43 2365-2373 (Preprint https://agupubs.onlinelibrary.wiley.com/doi/pdf/10.1002/2016GL068256) URL https://agupubs.onlinelibrary.wiley.com/doi/abs/10.1002/2016GL068256

[39] Sorokin D A, Tarasenko V F, Beloplotov D V and Lomaev M I 2019 Journal of Applied Physics 125 143301 (Preprint https://doi.org/10.1063/1.5067294) URL https://doi.org/10.1063/1. 5067294

[40] Briels T M P, van Veldhuizen E M and Ebert U 2008 IEEE Transactions on Plasma Science 36 908-909 ISSN 0093-3813

[41] Briels T M P, van Veldhuizen E M and Ebert U 2008 Journal of Physics D: Applied Physics 41 234008 URL https://doi.org/10.1088\%2F0022-3727\%2F41\%2F 23\%2F 234008

[42] Ebert U, Nijdam S, Li C, Luque A, Briels T and van Veldhuizen E 2010 Journal of Geophysical Research: Space Physics 115 A00E43 (Preprint https://agupubs.onlinelibrary.wiley. com/doi/pdf/10.1029/2009JA014867) URL https://agupubs.onlinelibrary.wiley.com/ doi/abs/10.1029/2009JA014867

[43] Sosnin E A, Naidis G V, Tarasenko V F, Skakun V S, Panarin V A, Babaeva N Y, Baksht E K and Kuznetsov V S 2018 Physics of Plasmas 25083513 (Preprint https://doi.org/10.1063/ 1.5038099) URL https://doi.org/10.1063/1.5038099

[44] Höft H, Becker M M and Kettlitz M 2018 Plasma Sources Science and Technology 27 03LT01 URL https://doi.org/10.1088\%2F1361-6595\%2Faab39d

[45] Böhm P, Kettlitz M, Brandenburg R, Höft H and Czarnetzki U 2016 Plasma Sources Science and Technology 25054002 URL https://doi.org/10.1088\%2F0963-0252\%2F25\%2F5\%2F054002

[46] Dogariu A, Goldberg B M, O'Byrne S and Miles R B 2017 Phys. Rev. Applied 7(2) 024024 URL https://link.aps.org/doi/10.1103/PhysRevApplied.7.024024

[47] Chng T L, Orel I S, Starikovskaia S M and Adamovich I V 2019 Plasma Sources Science and Technology 28045004 URL https://doi.org/10.1088\%2F1361-6595\%2Fab0b22

[48] Obrusník A, Bílek P, Hoder T, Šimek M and Bonaventura Z 2018 Plasma Sources Science and Technology 27085013 URL https://doi.org/10.1088\%2F1361-6595\%2Faad663

[49] Bílek P, Obrusník A, Hoder T, Šimek M and Bonaventura Z 2018 Plasma Sources Science and Technology 27085012 URL https://doi.org/10.1088\%2F1361-6595\%2Faad666

[50] Hoder T, Synek P, Chorvat D, Rahel J, Brandenburg R and Cernak M 2017 Plasma Physics and Controlled Fusion 59074001 URL http://stacks.iop.org/0741-3335/59/i=7/a=074001

[51] Bílek P, Šimek M and Bonaventura Z 2019 Plasma Sources Science and Technology 28115011 URL https://doi.org/10.1088\%2F1361-6595\%2Fab3936

[52] Wescott E M, Sentman D D, Heavner M J, Hampton D L, Osborne D L and Vaughan Jr O H 1996 Geophysical Research Letters 23 2153-2156 (Preprint https://agupubs . onlinelibrary. wiley.com/doi/pdf/10.1029/96GL01969) URL https://agupubs.onlinelibrary.wiley. com/doi/abs/10.1029/96GL01969

[53] Wescott E M, Sentman D D, Stenbaek-Nielsen H C, Huet P, Heavner M J and Moudry 
D R 2001 Journal of Geophysical Research: Space Physics 106 21549-21554 (Preprint https://agupubs.onlinelibrary.wiley.com/doi/pdf/10.1029/2000JA000429) URL https://agupubs.onlinelibrary.wiley.com/doi/abs/10.1029/2000JA000429

[54] Liu N and Pasko V P 2004 Journal of Geophysical Research: Space Physics 109 (Preprint https://agupubs.onlinelibrary.wiley.com/doi/pdf/10.1029/2003JA010064) URL https://agupubs.onlinelibrary.wiley.com/doi/abs/10.1029/2003JA010064

[55] Pipa A V, Koskulics J, Brandenburg R and Hoder T 2012 Review of Scientific Instruments 83 115112 ISSN 0034-6748

[56] Rabie M and Franck C M 2016 Journal of Physics D: Applied Physics 49175202 URL https: //doi.org/10.1088\%2F0022-3727\%2F49\%2F $17 \% 2$ F 175202

[57] Wormeester G, Pancheshnyi S, Luque A, Nijdam S and Ebert U 2010 Journal of Physics D: Applied Physics 43505201 URL https://doi.org/10.1088\%2F0022-3727\%2F43\%2F50\%2F505201

[58] Pancheshnyi S, Nudnova M and Starikovskii A 2005 Phys. Rev. E 71(1) 016407 URL https: //link.aps.org/doi/10.1103/PhysRevE.71.016407

[59] Šimek M and Ambrico P F 2012 Plasma Sources Science and Technology 21055014 URL https://doi.org/10.1088\%2F0963-0252\%2F21\%2F5\%2F055014

[60] Kulikovsky A A 1998 Phys. Rev. E 57(6) 7066-7074 URL https://link.aps.org/doi/10.1103/ PhysRevE.57.7066

[61] Donko Z, Schulze J, Mueller S and Czarnetzki U 2011 Applied Physics Letters 98251502 (Preprint https://doi.org/10.1063/1.3601486) URL https://doi.org/10.1063/1.3601486

[62] Hagelaar G J M and Pitchford L C 2005 Plasma Sources Science and Technology 14 722-733 URL https://doi.org/10.1088\%2F0963-0252\%2F 14\%2F4\%2F011

[63] BOLSIG+ solver ver 03/2016 Biagi database (N2,O2) www.lxcat.net online; retrieved on April 6,2017

[64] Šimek M 2014 Journal of Physics D: Applied Physics 47463001 URL https ://doi.org/10.1088\% $2 \mathrm{~F} 0022-3727 \% 2 \mathrm{~F} 47 \% 2 \mathrm{~F} 46 \% 2 \mathrm{~F} 463001$

[65] Gordillo-Vázquez F J, Passas M, Luque A, Sánchez J, van der Velde O A and Montanyá J 2018 Journal of Geophysical Research: Atmospheres 123 2336-2346 (Preprint https://agupubs.onlinelibrary.wiley.com/doi/pdf/10.1002/2017JD028126) URL https://agupubs .onlinelibrary.wiley.com/doi/abs/10.1002/2017JD028126

[66] Kanmae T, Stenbaek-Nielsen H C and McHarg M G 2007 Geophysical Research Letters 34 (Preprint https://agupubs.onlinelibrary.wiley.com/doi/pdf/10.1029/2006GL028608) URL https://agupubs .onlinelibrary.wiley.com/doi/abs/10.1029/2006GL028608

[67] Ihaddadene M A and Celestin S 2017 Journal of Geophysical Research: Space Physics 122 1000-1014 (Preprint https://agupubs.onlinelibrary.wiley.com/doi/pdf/10.1002/ 2016JA023111) URL https://agupubs.onlinelibrary.wiley.com/doi/abs/10.1002/ 2016 JA023111

[68] Neubert T, Østgaard N, Reglero V, Blanc E, Chanrion O, Oxborrow C A, Orr A, Tacconi M, Hartnack O and Bhanderi D D V 2019 Space Science Reviews 21526 ISSN 1572-9672 URL https://doi.org/10.1007/s11214-019-0592-z

[69] Lefeuvre F, Blanc E, Pinçon J L, Roussel-Dupré R, Lawrence D, Sauvaud J A, Rauch J L, de Feraudy H and Lagoutte D 2008 Space Science Reviews 137 301-315 ISSN 1572-9672 URL https://doi.org/10.1007/s11214-008-9414-4

[70] Neubert T, Østgaard N, Reglero V, Chanrion O, Heumesser M, Dimitriadou K, Christiansen F, Budtz-Jørgensen C, Kuvvetli I, Rasmussen I L, Mezentsev A, Marisaldi M, Ullaland K, Genov G, Yang S, Kochkin P, Navarro-Gonzalez J, Connell P H and Eyles C J 2020 Science 367 183-186 ISSN 0036-8075 (Preprint https://science.sciencemag.org/content/367/6474/183.full. pdf) URL https://science.sciencemag.org/content/367/6474/183 